\title{
QCLAS Sensor for Purity Monitoring in Medical Gas Supply Lines
}

\author{
Henrik Zimmermann ${ }^{1}$, Mathias Wiese ${ }^{1}$, Alessandro Ragnoni ${ }^{2}$ \\ ${ }^{1}$ neoplas control GmbH, Walther-Rathenau-Str. 49a, 17489 Greifswald, Germany \\ ${ }^{2}$ Loccioni, Via Fiume 16, 60030 Angeli di Rosora, Ancona, Italy
}

\begin{abstract}
Because of their direct impact on patience, medical supply lines underlie strict regulations and have to be monitored in terms of purity on regular base. State-of-the-art measurement solutions do not allow continuous bed-side monitoring. The aim of the presented project work is to provide a compact multispecies monitoring system based on latest quantum-cascade-laser technologies.
\end{abstract}

Keywords: gas-sensor technology, optical measurement methodology, quantum-cascade-laser, laser absorption spectroscopy, purity measurements

\section{Introduction}

The neoplas control $\mathrm{GmbH}$ is a spin-off of the INP Greifswald (Leibniz-Institute for Plasma Research and Technology). The solutions offered are customized devices and product specific services for gas and plasma diagnostics in industrial as well as economic environments. While implementing this business idea, close cooperation with both the INP Greifswald and its transfer center, the neoplas $\mathrm{GmbH}$, are maintained.

The products of the Q-MACS family (Quantum Cascade Laser Measurement and Control System) are compact and user friendly. The Quantum cascade laser measurement and control systems have been developed to monitor and control plasma processes and to detect highly sensitive trace gases [1]. Q-MACS combine the advantages of absorption spectroscopy in the mid infrared spectral range with the unique properties of Quantum Cascade Lasers (QCL). In combination with thermo-electric cooled infrared detectors Q-MACS is well suited for industrial applications, in particular for on-line process monitoring. The Q-MACS products need almost no maintenance and can be individually adapted to customer requirements inside industrial processes due to its modular design. With the product series Q-MACS it is possible to detect lowest absolute concentrations of molecules in gaseous media up to parts per billion ( $p p b)$ in real-time.

Within the scope of the EU funded project MIRIFISENS the issues of sensitivity and selectivity, multi-gas capabilities, compactness, efficiency and cost effectiveness as specified by a number of selected safety and security applications are addressed [2]. In this context the neoplas control $\mathrm{GmbH}$ in cooperation with the project partner Loccioni is focusing on the on-site purity monitoring of medical gases used in hospitals. The main 3 supply lines to check are the oxygen line, the nitrogen protoxide line and the compressed air line. Continuous monitoring on the distribution lines could avoid fatal accidents that unfortunately still happen to patients, especially after maintenance on older buildings. Concentration limits are defined for every gas line by local regulatory. The main molecules to be measured are $\mathrm{CO}_{2}, \mathrm{CO}, \mathrm{H}_{2} \mathrm{~S}$, $\mathrm{SO}_{2}, \mathrm{NO}_{x}, \mathrm{H}_{2} \mathrm{O}, \mathrm{O}_{2}, \mathrm{~N}_{2} \mathrm{O}$ and Oil. Expensive instrumentation has been developed to perform periodical check to every gas port, but these systems do not allow the bed site operation. Therewith a compact lower cost sensing technology ensures higher safety potential as it could be directly integrated in the distribution plant. As a first step a single species monitor focusing on the challenging high sensitive detection of $\mathrm{SO}_{2}$ is developed and forms the basis for the intended multispecies solution based on MIRIFISENS technology.

\section{Experimental setup}

As a laser absorption spectrometer based on direct absorption technique the Q-MACS Trace compact $\mathrm{SO}_{2}$ sensor acquires the transmission signal after passing the $56 \mathrm{~m}$ long path cell. This signal will be analyzed with respect to well-known molecular parameters, what ensures almost calibration free 
measurements. In terms of the introduced $\mathrm{SO}_{2}$ measurements these parameters are taken from the HITRAN molecular absorption database [3]. In Figure 1a a representative detector signal acquired at the chosen Full Scale value of $7 \mathrm{ppm}$ and the corresponding fit result is shown. The scheme of the experimental setup is shown in Fehler! Verweisquelle konnte nicht gefunden werden.b.

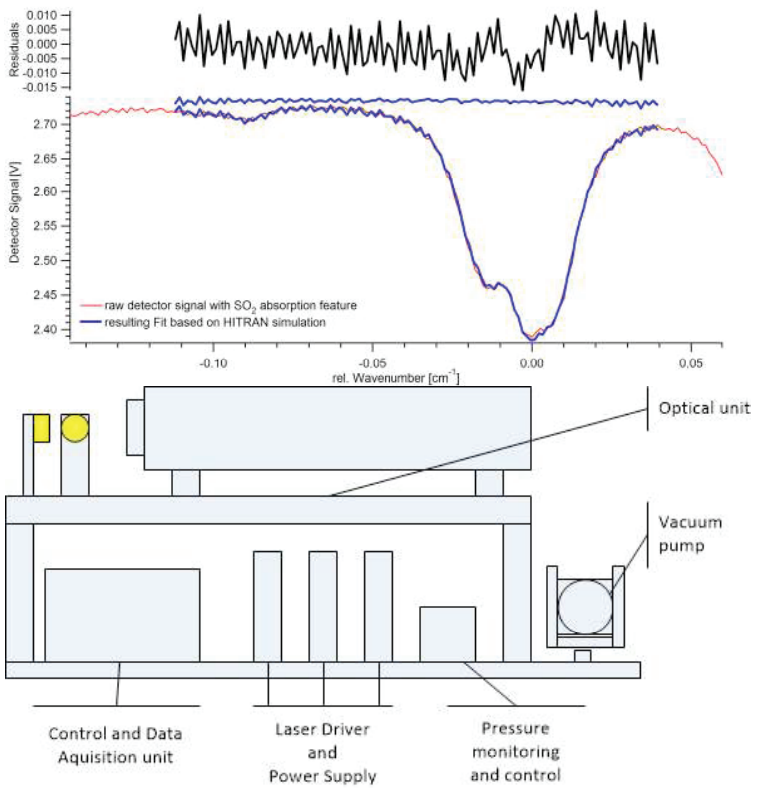

Figure 1: (a) Acquired detector signal with $\mathrm{SO}_{2}$ absorption feature and fit result. (b) Experimental configuration.

The gas dilution system consisted of two Unit MKS MF1 mass flow controllers and a diaphragm pump Thomas 70112 DC. One controller provides a range from 0 to $200 \mathrm{sccm}$ and another one comes with a range from 0 to $10 \mathrm{slm}$. This set of flow controllers allows reasonable accuracy for the dilution of gas standards over a very wide range of dilution ratios by selecting the appropriate settings on the mass flow controllers. The distinct concentration steps, which were alternatingly tuned as continuous flow through the measurement cell of 0.5 I volume, are reflected in Table 1.

Table 1: List of dilution steps and resulting $\mathrm{SO}_{2}$ mixing ratios and achievable accuracy

\begin{tabular}{|c|c|c|c|}
\hline $\begin{array}{c}\text { Target / } \\
\mathrm{ppb}\end{array}$ & $\begin{array}{c}\mathrm{SO}_{2} / \\
\text { sccm }\end{array}$ & $\begin{array}{c}\mathrm{N}_{2} / \\
\text { sccm }\end{array}$ & $\begin{array}{c}\text { Accuracy / } \\
\mathrm{ppb}\end{array}$ \\
\hline 0 & 0 & 300 & $+15 /-0$ \\
\hline 70 & 2 & 298 & $+23 /-19$ \\
\hline 170 & 5 & 295 & $+35 /-30$ \\
\hline 350 & 10 & 290 & $+55 /-47$ \\
\hline 690 & 20 & 280 & $+94 /-80$ \\
\hline 1700 & 50 & 250 & $+209 /-180$ \\
\hline 3500 & 100 & 200 & $+395 /-344$ \\
\hline
\end{tabular}

\begin{tabular}{|l|l|l|l|}
\hline 5200 & 150 & 150 & $+574 /-502$ \\
\hline 7000 & 200 & 100 & $+747 /-655$ \\
\hline
\end{tabular}

This dilution system was used to provide the test atmospheres for the analyzers under test.

The pressure in the sample cell was monitored via a MKS HPS Series 902 piezo transducer, which provides a range of application from 0 to 1300 mbar. Using this pressure gauge the pressure in the cell was ensured to be a fixed value of $\sim 60$ mbar during the tests. An active pressure control was not applied during this test.

\section{Statistical Methods}

The statistical methods used to evaluate the quantitative performance factors are presented in this chapter. Because no alternative concept for the detection of $\mathrm{SO}_{2}$ was available during the test, the evaluation of the performance parameters for the sensor has to be based on the calculated mixing ratios, what depend on the settled flow rates. This approach limits the types of statistical comparisons, which could be applied. Qualitative observations were also used to evaluate verification test data.

Linearity factor is assessed by linear regression with the calibration concentration as the independent variable and the analyzer response as the dependent variable. The calibration model is given in Eq. (1).

$$
Y_{C}=h(c)+\text { error }_{C}
$$

Where $Y_{C}$ is the analyzer's response to a challenge concentration $\mathrm{c}, \mathrm{h}(\mathrm{c})$ is a linear calibration curve, and the error term is assumed to be normally distributed. Variability $\sigma$ of the measured concentration values $c$ was modelled by the relationship expressed in Eq. (2).

$$
\sigma_{C}^{2}=\alpha+k c^{\beta}
$$

Where $\alpha, k$ and $\beta$ are constants to be estimated from the data. After determining the relationship between the mean and variability, appropriate weighting is determined by Eq. (3).

$$
w_{C}=\frac{1}{\sigma_{C}^{2}}
$$

The form of the regression model to be fitted is expressed in Eq. (3).

$$
c=h^{-1}\left(Y_{C}\right)=\frac{\left(Y_{C}-\alpha_{0}\right)}{\alpha_{1}}
$$

Concentration values were calculated from the estimated calibration curve using the formula Eq. (4).

$$
h(c)=\alpha_{0}+\alpha_{1} \cdot c
$$

A test for departure from linearity is carried out by comparing the residual sum of squares to a chi-square distribution with $6-2=4$ degrees of freedom, as given with Eq. (5). 


$$
\sum_{i=1}^{6}\left(\bar{Y}_{C_{i}}-\alpha_{0}-\alpha_{1} c_{i}\right)^{2} \cdot n_{C_{i}} \cdot w_{C_{i}}
$$

Where $\mathrm{n}_{\mathrm{C}}$ is the number of replicates at concentration $\mathrm{c}$.

The response time of the analyzers to a step change in analyte concentration was calculated by determining the total change in response due to the step change (either increase or decrease) in concentration, and then determining the point in time when $95 \%$ of that change was achieved. Both rise and fall times were determined. Using data taken every 1 second, the calculation is carried out by Eq. (6).

$$
R_{\text {Total }}=R_{a}-R_{b}
$$

Where $R_{a}$ is the final response of the analyzer to the test gas after the step change, and $R_{b}$ is the final response of the analyzer before the step change. The analyzer response that indicates the response time then is calculated applying Eq. (7).

$$
R=0.95 \cdot R_{\text {Total }}
$$

The point in time at which this response occurs is determined by inspecting the response time data, and the response time is calculated according to Eq. (8).

$$
\text { Time } e_{\text {Response }}=\text { Time }_{95 \%}-\text { Time }_{I}
$$

Where Time $_{95 \%}$ is the time at which R occurs, and Time, is the time at which the step change in concentration was imposed. Since only one determination was made, the precision of the rise and fall time results could not be estimated.

The detection limit (LOD) was defined as the smallest true concentration at which the analyzers expected response exceeded the calibration curve at zero concentration by three times the standard deviation of the analyzers zero reading. The LOD is then determined by applying Eq. (9).

$$
L O D=\frac{\left[\left(\alpha_{0}+3 \cdot \sigma_{0}\right)-\alpha_{0}\right]}{\alpha_{1}}=\frac{3 \cdot \sigma_{0}}{\alpha_{1}}
$$

Here $\sigma_{0}$ is the estimated standard deviation at zero concentration. Note that the validity of the detection limit estimate and its standard error depends on the validity of the assumption that the fitted linear calibration model accurately represents the response down to zero concentration.

Statistical procedures for assessing zero and span drift were similar to those used to assess interrupted sampling. Zero (span) drift was calculated as the arithmetic difference between zero and span values respectively obtained before and after sampling of source emissions. During this test no estimate of the precision of the zero and span drift values was made.

\section{Results and Discussion}

A Q-MACS Trace analyzer prototype was tested for the highest sensitive online monitoring of $\mathrm{SO}_{2}$ traces. The laboratory tests were designed to challenge the analyzer over its full range under a variety of conditions. These tests were performed using certified standard gases and a gas dilution system. The gas standards were diluted with high-purity gases to produce the desired range of concentrations with known accuracy. Laboratory testing was conducted primarily by supplying known gas mixtures to the Q-MACS Trace analyzer from the gas delivery system. The linearity of response of the Q-MACS Trace analyzer will be tested by 30-point calibrations of the $\mathrm{SO}_{2}$ gas filling. Prior to this check, the analyzer is provided with the appropriate zero gas $\left(\mathrm{N}_{2}\right)$ and then with a span gas concentration of $7 \mathrm{ppm} \mathrm{SO}_{2}$, which is defined in this verification test to be the nominal range of the analyzer. After any necessary adjustments to the analyzer to match that span value, the 30-point check proceeded without further adjustments. The 30 points consisted of three replicates each at $70 \mathrm{ppb}, 170 \mathrm{ppb}$, 350 ppb, 690 ppb, 1.7 ppm, 3.5 ppm, 5.2 ppm and $7 \mathrm{ppm}$ in random order, and interspersed with six replicates of zero gas. Following completion of all 30 points, the zero and $100 \%$ spans were repeated, also without adjustment of the analyzer. Zero and span drift will be evaluated using data generated in the linearity and the accuracy tests. The zero and span drift is determined as the difference in response on zero and span gases in these two tests. This comparison will be made for all zero and span responses, using data from the linearity and the accuracy tests. Figure 2 shows the linearity results obtained from the linearity tests for the Q-MACS Trace analyzer, which was configured for the $\mathrm{SO}_{2}$ measurements.

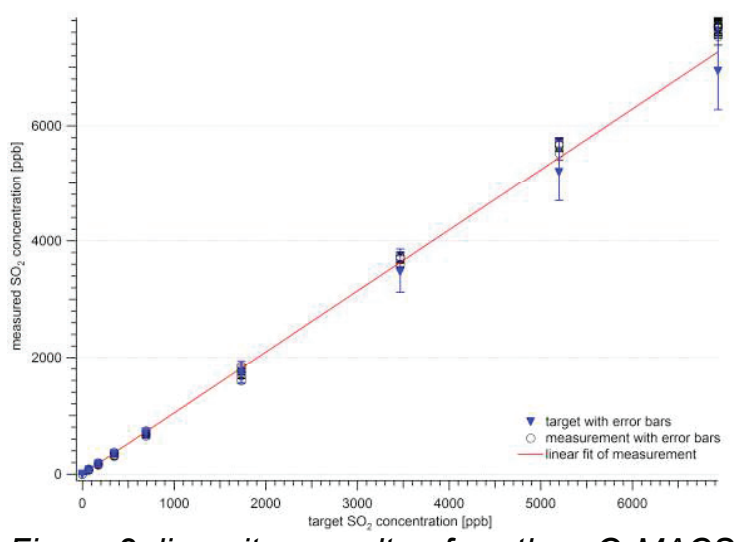

Figure 2: linearity results for the Q-MACS Trace set up with 1 second acquisition time.

In Table 2 the linear equations for the system configuration developed from this data is shown. 
Table 2: Statistic results of the linearity test.

\begin{tabular}{|c|c|c|}
\hline Intercept / ppb & Slope & $R^{2}$ \\
\hline$-0.271 \pm 3.74$ & $1.048 \pm 0.02$ & 0.823 \\
\hline
\end{tabular}

The quite low value for the regression coefficient in the linearity tests is caused by the rather high uncertainty the chosen dilution system provides for the $\mathrm{N}_{2}$ dilution gas at lower flow rates. The uncertainties of the dilution system are listed in Table 1 as well as visualized in terms of error bars in Figure 2. For the linear fit the concentration values are weighted with the uncertainties of the dilution system to respect the deviation of the finally settled concentrations from the targeted ones.

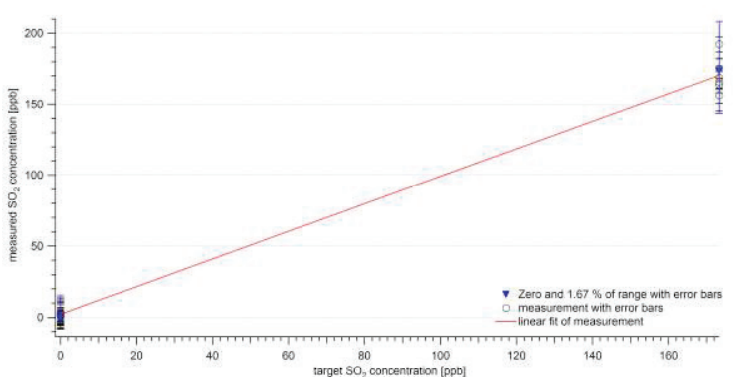

Figure 3: results of linear fit for measured values at Zero and $1.67 \%$ of range.

Table 3: Detection limit determined according to $\mathrm{SO}_{2}$ measurements.

\begin{tabular}{|l|c|}
\hline Slope & $0.969 \pm 0.086$ \\
\hline $\begin{array}{l}\text { Standard Deviation } \\
\text { for Zero values / ppb }\end{array}$ & 4.26 \\
\hline Average Noise / ppb & 7.67 \\
\hline Detection Limit / ppb & 22.99 \\
\hline
\end{tabular}

Table 3 shows the detection limits for each configuration of the Q-MACS Trace analyzer tested, determined from the detection limit procedure described in the former section. Figure 3 visualizes the results for the linear fit valid for the range from Zero to $1.67 \%$ of range. The calculated detection limit for 1 second acquisition time is $22.99 \mathrm{ppb}$.

The response time results for the sensor based on a step change in analyte concentration was determined to about $14 \pm 1 \mathrm{~s}$, when limiting to an acquisition time of 1 second.

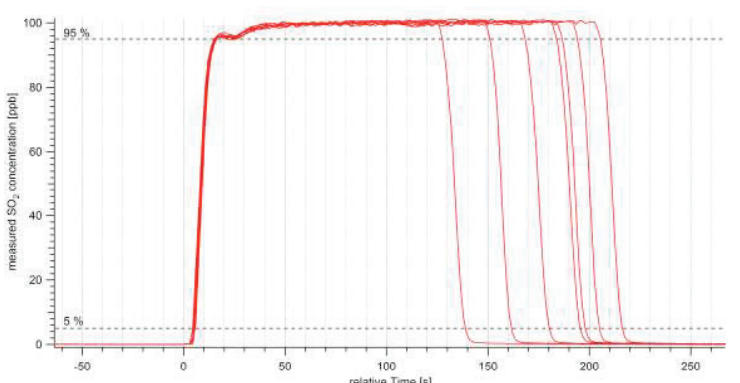

Figure 4: trend of $\mathrm{SO}_{2}$ concentration during the zero / span test.
In Figure 4 the trend of the $\mathrm{SO}_{2}$ concentration signal is shown.

Zero and span data taken at the start and end of the linearity test are shown in Table 4. The drift values observed are shown in Table 5 as differences between the pre- and post-test concentration measurements. Furthermore, Table 5 also presents the zero and span drifts as a percent of span gas concentrations. The Zero drift for the sensor tested was less than $0.04 \%$ of the respective span gas concentration. The span drift was less than $0.22 \%$ of the respective span concentration.

Table 4: Data used to assess Zero and Span drift of the Q-MACS Trace compact $\mathrm{SO}_{2}$ analyzer.

\begin{tabular}{|l|c|}
\hline \multicolumn{1}{|c|}{ Component } & Sensor SO2 / ppb \\
\hline Pre-Test Zero & $0.64 \pm 8.48$ \\
\hline Pre-Test Span & $7674.57 \pm 128.13$ \\
\hline Post-Test Zero & $3.13 \pm 7.40$ \\
\hline Post-Test Span & $7689.83 \pm 161.16$ \\
\hline
\end{tabular}

Table 5: Results of the Zero and Span drift of the $\mathrm{SO}_{2}$ analyzer.

\begin{tabular}{|l|c|}
\hline \multicolumn{1}{|c|}{ Component } & Difference / ppb \\
\hline Zero & 2.49 \\
\hline Span & 15.26 \\
\hline Drift in \% of Span \\
\hline - Zero & $0.04 \%$ \\
\hline - Span & $0.22 \%$ \\
\hline
\end{tabular}

The final verification tests were conducted with support of the Leibniz-Institute for Plasma Science and Technology Greifswald.

\section{Summary and Conclusions}

The test results, which are summarized in Table 6, confirm that the Q-MACS Trace analyzer provides linear response over wide operating ranges. The compact prototype configuration as used in this preliminary study provides very good results with respect to the sensitivity, selectivity and stability.

Table 6: Results from performance analysis for the Q-MACS Trace $\mathrm{SO}_{2}$ Sensor.

\begin{tabular}{|l|c|}
\hline \multicolumn{1}{|c|}{ Specification } & Value \\
\hline Response time & $14 \pm 1 \mathrm{~s}$ \\
\hline Linearity & 1.048 \\
\hline Detection limit & $>23 \mathrm{ppb}$ \\
\hline Drift & \\
\hline Zero & $<0.04 \%$ \\
\hline Span & $<0.22 \%$ \\
\hline
\end{tabular}

The system is rugged and portable, and the necessary setup time is minimal. The quite fast sensor response times and measurement stability allowed verification testing to proceed smoothly. Its design incorporates a sample 
probe and sample conditioning system, making it adaptable to a wide range of measurement applications.

Although the aim of $15 \mathrm{ppb}$ and below for the limit of detection was not achieved with this Q-MACS Trace compact configuration, the positive results show that it is possible to design a system, which will fulfil this specification. Recently long path cells with more than 150 meters optical path length became available. Unfortunately such cell would not allow keeping or even further optimizing the compactness of the resulting sensor system. Moreover, the much higher volume of such a cell would lead to a significant and unwanted increase of the response time. More practical approaches are the selection of stronger absorption lines, what could be possible depending on the gas matrix to be analyzed in the specific application. Developments for further optimization of the gas handling, e.g. by the integration of an active pressure control, as well as the extension of the analytical methods are actually in progress. Applying these drafted optimizations in a future configuration will allow decreasing the achievable detection limit by approx. a factor of 5 and therefore to detect $\mathrm{SO}_{2}$ concentrations below $5 \mathrm{ppb}$ by a continuous monitoring system.

In pending development steps the single QCL source will be exchanged by a QCL to address several molecular species in parallel by maintaining the compactness of the system. First introduced by Lee et al. in 2009 [5], QCL arrays will now make the step from the lab to the market. This was made possible in the rather short timeframe thanks to funded research and development projects like MIRIFISENS.

\section{References}

[1] J. Röpcke, et al., J. Phys. D: Applied Phys. 45, 10pp (2012); doi: 10.1088/00223727/45/42/423001

[2] Mid InfraRed Innovative lasers For Improved SENSor of hazardous substances, FP7-ICT-2011.3.5 Core and disruptive photonic technologies, http://www.mirifisens-project.eu.

[3] L.S. Rothman, et al., Journal of Quantitative Spectroscopy and Radiative Transfer 130, 4-50 (2013); doi: 10.1016/j.jqsit.2013.07.002

[4] M. Fischer, et al., Optics Express 22, 70147027 (2014); doi: 10.1364/OE.22.007014

[5] B. G. Lee, et al., Optics Express 17, 1621616224 (2009); doi: 10.1364/OE.17.016216 